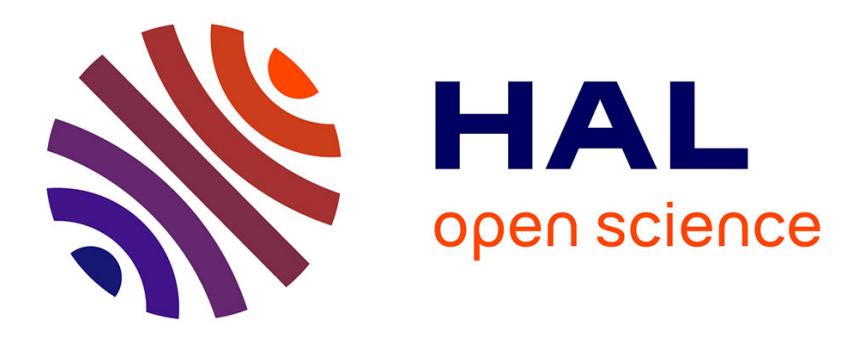

\title{
Résistivité électrique du bismuth irradié aux neutrons rapides
}

\author{
G. Quelard
}

\section{To cite this version:}

G. Quelard. Résistivité électrique du bismuth irradié aux neutrons rapides. Journal de Physique Lettres, 1975, 36 (10), pp.247-248. 10.1051/jphyslet:019750036010024700 . jpa-00231199

\section{HAL Id: jpa-00231199 https://hal.science/jpa-00231199}

Submitted on 1 Jan 1975

HAL is a multi-disciplinary open access archive for the deposit and dissemination of scientific research documents, whether they are published or not. The documents may come from teaching and research institutions in France or abroad, or from public or private research centers.
L'archive ouverte pluridisciplinaire HAL, est destinée au dépôt et à la diffusion de documents scientifiques de niveau recherche, publiés ou non, émanant des établissements d'enseignement et de recherche français ou étrangers, des laboratoires publics ou privés. 
Classification

Physics Abstracts

7.184

\title{
RÉSISTIVITÉ ÉLECTRIQUE DU BISMUTH IRRADIÉ AUX NEUTRONS RAPIDES
}

\author{
G. QUELARD \\ Section d'Etude des Solides Irradiés, C.E.N., 92260 Fontenay-aux-Roses, France
}

(Reçu le 24 avril 1975, révisé le 19 juin 1975, accepté le 24 juin 1975)

Résumé. - Nous avons irradié du bismuth, à $20 \mathrm{~K}$, aux neutrons rapides. Les variations de résistivité électrique, au cours de l'endommagement et du recuit, sont indépendantes de l'orientation cristallographique et indiquent une création de porteurs au cours de l'irradiation.

\begin{abstract}
The production and recovery of fast neutron radiation damage in bismuth, at $20 \mathrm{~K}$ has been studied by means of electrical resistivity. Results are independent of crystallographic orientation and indicate a creation of carriers during irradiation.
\end{abstract}

Dans le but de mieux connaitre les propriétés électroniques du bismuth de nombreux auteurs étudient l'influence des défauts sur ces propriétés; effet des impuretés [1, 2, 3]; effet de trempe [4]. Pour notre part nous nous intéressons plus directement aux défauts cristallins et nous présentons ici les résultats obtenus sur la résistivité électrique du bismuth irradié aux neutrons rapides à $20 \mathrm{~K} \mathrm{[5]} \mathrm{dans} \mathrm{la} \mathrm{pile} \mathrm{Triton}$ du C.E.N. de Fontenay-aux-Roses.

Nous avons irradié successivement des échantillons polycristallins, fils de section $1 \mathrm{~mm}^{2}$, avec un flux instantané de $4 \times 10^{11}$ neutrons rapides $\mathrm{cm}^{-2} \mathrm{~s}^{-1}$, et des échantillons monocristallins, de section $10 \mathrm{~mm}^{2}$, avec un flux instantané de $8 \times 10^{10}$ neutrons rapides $\mathrm{cm}^{-2} \mathrm{~s}^{-1}$. La pureté des échantillons est de $6 \mathrm{~N}$ ou 99,999 9\% (origine : Société Braconnot). L'étude du groupe de symétrie $3 \overline{\mathrm{m}}$ du bismuth [6] montre que le tenseur de conductivité ne comporte que deux éléments indépendants. Nous avons donc choisi deux monocristaux d'orientation $\langle 111\rangle$ et perpendiculaire à $\langle 111\rangle$ suivant leur dimension la plus longue.

Les résistivités du bismuth selon les deux directions des monocristaux montrent une faible anisotropie (quelques \% à $20 \mathrm{~K}$ ), qui ne rend pas compte de la forte anisotropie des tenseurs de mobilités $\boldsymbol{\mu}$ et $\boldsymbol{v}$ des électrons et des trous [7] :

$$
\sigma=\text { nep }+ \text { pev. }
$$

Nous avons mesuré à $20 \mathrm{~K}$ les variations de résistivité des échantillons au cours de l'endommagement (Fig. 1) et au cours de la guérison de cet endommagement par recuits isochrones de $10 \mathrm{~min}$. depuis la température d'irradiation $(20 \mathrm{~K})$ jusque vers $400 \mathrm{~K}$ (Fig. 2).

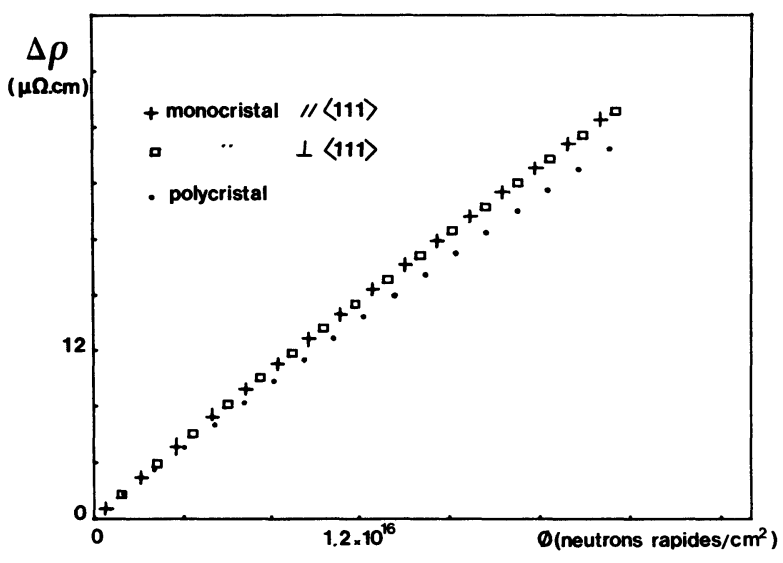

Fig. 1. - Variations de résistivité du bismuth en fonction de la fluence. Irradiation à $20 \mathrm{~K}$. 1) monocristaux $(+, \square)$ irradiés avec un flux de $\left.8 \times 10^{10} \mathrm{n} \mathrm{cm}^{-2} \mathrm{~s}^{-1} ; 2\right)$ polycristal (.) irradié avec un flux de $4 \times 10^{11} \mathrm{n} \mathrm{cm}^{-2} \mathrm{~s}^{-1}$.

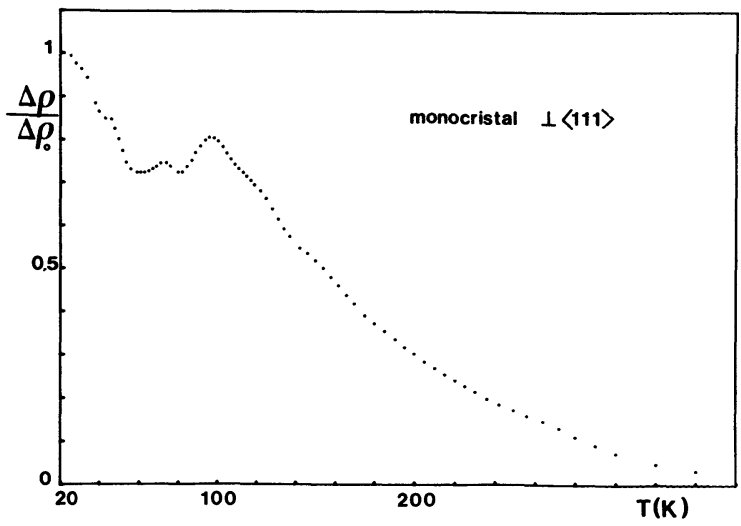

FIG. 2. - Restauration de la résistivité du bismuth par recuits isochrones après irradiation à une fluence de $2,2 \times 10^{16} \mathrm{n} \mathrm{cm}^{-2}$. 
Sur la figure 1, on remarque que, compte tenu des erreurs expérimentales dues à l'imprécision sur le flux instantané, les résultats sont indépendants de l'orientation cristallographique. Ce fait est inattendu si l'on considère la présence de deux types de porteurs avec des tenseurs de mobilités différents et anisotropes; la résistivité intrinsèque du défaut n'a alors pas de raison d'être isotrope. Des expériences [4] montrent d'ailleurs une anisotropie de la résistivité intrinsèque des défauts obtenus par trempe. Par comparaison avec un métal on peut faire deux remarques sur le comportement du bismuth : l'augmentation de résistivité est très forte : 2 ordres de grandeur supérieure à celle d'un métal pour des fluences comparables, et on observe une courbure négative, sur le graphe donnant l'endommagement en résistivité, dès les très faibles fluences.

Sur la figure 2 nous n'avons représenté qu'une seule courbe de recuits isochrones; les courbes pour un monocristal d'orientation $\langle 111\rangle$ et pour un polycristal sont rigoureusement identiques à la première. Nous ne pouvons pas encore attribuer les différents stades observés à la migration de tel ou tel défaut, interstitiel ou lacunaire. Il faut noter les remontées de résistivité, en particulier celles se situant vers 85-100 K. Dans un métal pur, pour continuer notre comparaison, on n'observe pas de telles remontées. On ne peut vraisemblablement les expliquer que par une brusque modification du nombre de porteurs.

$\mathrm{Au}$ cours de l'irradiation il y a donc une variation du nombre de porteurs. Avec nos résultats il est difficile de préciser cette variation. En effet :

- l'irradiation aux neutrons rapides produit des défauts différents : paires de Frenkel, défauts isolés et amas de défauts,

- on ne connaît pas la nature électrique exacte des défauts créés,

- la présence de deux types de porteurs rend difficile la séparation entre les effets de mobilités et les effets de nombre de porteurs.

On peut donner un ordre de grandeur de la concentration globale de défauts créés par l'irradiation aux neutrons rapides en considérant celui obtenu pour les métaux (ex. : $\mathrm{Cu}$ ) : une irradiation de $10^{17}$ neutrons rapides $/ \mathrm{cm}^{2}$ donne une concentration globale de défauts de $10^{-4}$ environ.

Pour donner une explication qualitative et quantitative des résultats obtenus nous comptons irradier des échantillons dont nous modifions la répartition initiale des porteurs par dopage avec du tellure ou de l'étain. Nous envisageons également des irradiations aux électrons avec des mesures d'effet Hall.

\section{Bibliographie}

[1] Noothooven van Goor, J. M., Phillips Res. Rept. Suppl. (1971) 4.

[2] Bate, R. T. and Einspruch, N. G., Phys. Rev. 153 (1967) 796.

[3] Kao, Y. H., Brown III, R. D., Hartman, R. L., Phys. Rev. 136 (1964) 858.
[4] Saunders, G. A. and Sümengen, Z., Proc. R. Soc. A 329 (1972).

[5] Conte, R. R., Dural, J., Revue Phys. Appl. 2 (1967) 1.

[6] JURETSCHKe, H. J., Acta Crystallogr. 8 (1955) 716.

[7] Hartman, R., Phys. Rev. 181 (1969) 1070. 